\title{
Cosmic metal enrichment
}

\author{
Andrea Ferrara ${ }^{1}$ \\ ${ }^{1}$ SISSA/ISAS, Via Beirut 2-4, 34014 Trieste, Italy \\ email: ferrara@sissa.it
}

\begin{abstract}
I review the present understanding of the process by which the universe has been enriched in the course of its history with heavy elements produced by stars and transported into the surrounding intergalactic medium. This process goes under the name of "cosmic metal enrichment" and presents some of the most challenging puzzles in present day physical cosmology. These are reviewed along with some proposed explanations that all together form a coherent working scenario.
\end{abstract}

Keywords. (galaxies:) intergalactic medium, cosmology: theory, galaxies: high-redshift

\section{Preliminaries}

After the Big Bang the cosmic gas had a composition which was (virtually) free of heavy elements. Yet, every single parcel of gas that we can probe today with our most powerful telescopes shows the sign of considerable amounts of metals, up to the highest redshift. When these metals where first produced, by what sources, and how these atomic species traveled away from their production sites are among the most challenging puzzles in current cosmological scenarios.

A very simple, and yet robust, estimate of the amount of metals present at the end of reionization can be made. This is based on the fact that the sources of heavy elements and photons with energy $>1$ Ryd responsible for hydrogen reionization are massive stars. Hence, as we know that reionization was complete by redshift $z=6$, we can compute the metallicity of the cosmic gas associated with the ionizing photons required to reionize the universe, in the hypothesis that such process has been powered by stellar radiation. Obviously, as different type of sources, as quasars and/or decaying/annihilating dark matter particles may have contributed, the result of this simple exercise is an upper limit to the amount of metals. However, many arguments suggest that stars are by far the most viable reionization source candidates. In this case, we find that the mean metallicity of the cosmic gas (in practice, the intergalactic medium [IGM] which contains most of the baryons) at $z=6$ is

$$
Z=\frac{\langle y\rangle \nu C}{\eta}=3.5 \times 10^{-4} C Z_{\odot}
$$

where $\langle y\rangle$ is the mean supernova metal yield, $\nu$ is the number of supernovae per unit stellar mass formed, $C \approx 1-10$ is the IGM clumping factor and $\eta$ is the number of ionizing photons per baryon into stars. The above result has been derived for a Salpeter stellar IMF but the value of $Z$ is only mildly dependent on such quantity. The main point is that metal production associated with cosmic reionization is already substantial and comparable with the value derived from QSO absorption line experiments at lower redshifts.

The next question concerns the sources that predominantly produced the metals and photons. Current data from a number of different experiments including CMB polarization anisotropies, Ly $\alpha$ and Ly $\beta$ Gunn-Peterson tests, cosmic star formation history and 
galaxy number counts constrain reionization history in a very stringent way (Choudhury \& Ferrara 2007). Thus it is possible from those models to determine which sources were contributing most of the ionizing photons (and hence metals) at a given redshift by considering the fractional instantaneous contribution of halos above a certain mass,

$$
f_{\gamma}(>M, z) \equiv \frac{\dot{n}_{\gamma}(>M, z)}{\dot{n}_{\gamma}(z)},
$$

The main conclusion from that study is that $>80 \%$ of the ionizing power at $z \geqslant 7$ is provided by halos with masses $<10^{9} M_{\odot}$ which are predominantly harboring metal-free (PopIII) stars. A turnover to a PopII-dominated reionization phase occurs shortly after, with this population, residing in $M>10^{9} M_{\odot}$ haloes, producing $\approx 60 \%$ of the ionizing photons at $z=6$. In conclusion, PopIII stars and small galaxies initiate reionization at high redshift and remain important until they are overcome by PopII stars and QSOs below $z=7$.

It then appears that cosmic metal enrichment is a gradual process that proceeds along with the increase of the mean ionized fraction in the universe and that the heavy elements were predominantly expelled at high redshifts $(z>6)$ by dwarf-like galaxies. These galaxies had probably larger ejection efficiencies with respect to "normal" ones; in addition such early enrichment has interesting properties and implications that we discuss in the following.

\section{Early enrichment by dwarf galaxies}

Mechanical feedback results from the energy deposited in the surrounding medium by winds from massive stars and supernova explosions. The powerful shocks originating from such events heat and accelerate the gas, driving outflows of heavily metal-enriched gas into the IGM. The central question of the problem concerns the fraction of produced metals that escape from the galaxy, i.e. the metal escape fraction, $\delta_{B}$. A solution has been proposed by Ferrara, Pettini \& Shchekinov (2000) who noticed that SNe in "normal" galaxies (as for example the Milky Way) are distributed in the disk and clustered in OB associations; thus, these explosion sites act incoherently. In low-mass galaxies, on the contrary, the size of the galaxy is comparable to the size of individual SN-driven bubbles and therefore the energy deposited can work to drive coherently the same outflow. This makes the metal escape fraction from the latter objects much larger than from large galaxies. To see this point quantitatively, we recall that in nearby galaxies it is found that the luminosity function of $\mathrm{OB}$ associations is well approximated by a power-law,

$$
\phi(N)=\frac{d \mathcal{N}_{O B}}{d N}=A N^{-\beta},
$$

with $\beta \approx 2$. Here $\mathcal{N}_{O B}$ is the number of associations containing $N$ OB stars; normalization of $\phi(N)$ to unity requires $A=1$. Thus the probability for a cluster of OB stars to host $N$ SNe is $\propto N^{-2}$, where $N=L_{O B} t_{O B} / \epsilon_{0}$, and $t_{O B}=40 \mathrm{Myr}$ is the time at which the lowest mass $\left(\approx 8 M_{\odot}\right)$ SN progenitors expire. The total mechanical luminosity, is then found to be

$$
L_{t}(z)=\int_{N_{m}}^{N_{M}} L_{O B}(N) \phi d N
$$


where $N_{m}=1\left(N_{M}\right)$ is the minimum (maximum) possible number of SNe in an association. This gives

$$
L_{t}(z)=\text { const. } \frac{\epsilon_{0}}{t_{O B}} \ln \frac{N_{M}}{N_{m}} .
$$

The contribution to the total luminosity from clusters powerful enough to lead to blowout is

$$
L_{B}\left(z,>L_{c}\right)=\text { const. } \frac{\epsilon_{0}}{t_{O B}} \ln \frac{N_{M}}{N_{c}},
$$

where $N_{c}$ is the number of $\mathrm{SNe}$ in a cluster with mechanical luminosity equal to $L_{c}$, i.e.

$$
N_{c}=\frac{L_{c} t_{O B}}{\epsilon_{0}} .
$$

Thus, the fraction of mechanical energy (and metals) that can be blown out is

$$
\delta_{B}=\frac{\ln \left(N_{M} / N_{c}\right)}{\ln \left(N_{M} / N_{m}\right)}<1 .
$$

Clearly, $N_{M}$ (and therefore $\delta_{B}$ ) is an intrinsically stochastic number. To determine its dependence on the total number of supernovae $N_{t}=L_{t}(z) t_{O B} / \epsilon_{0}$ produced by a galaxy during the lifetime of an OB association, we have used a Monte Carlo procedure applied to the distribution function in eq. 2.1. For low values of $N_{t}$ the quantity $N_{M}$ is larger than $N_{c}$, implying that in every galaxy at least some superbubbles are able to blow out. However, near $N_{t}=10^{4} N_{M}$ flattens and eventually becomes equal to $N_{c}$ at $N_{t} \simeq$ 45000 . Above this limit (corresponding to a galaxy with $\dot{M}_{\star} \approx 0.35 M_{\odot} \mathrm{yr}^{-1}$ or $M_{h} \approx$ $\left.10^{12}(1+z)^{-3 / 2} M_{\odot}\right)$ blowout is inhibited. The fraction $\delta_{B}$ is a decreasing function of $N_{t}$; an approximate analytical form is

$$
\begin{array}{r}
\delta_{B}\left(N_{t}\right)=1 \text { for } N_{t}<100 \\
\delta_{B}\left(N_{t}\right)=a+b \ln \left(N_{t}^{-1}\right) \text { for } N_{t}>100,
\end{array}
$$

with $a=1.76, b=0.165$. Clearly, in small galaxies even the smallest associations are capable of producing blowout, and therefore $\delta_{B} \rightarrow 1$, whereas for large galaxies there are less and less $\mathrm{OB}$ associations able to vent their metals and hot gas into the IGM, i.e. $\delta_{B} \rightarrow 0$. The transition occurs roughly when the mass of the galaxy is $\approx 1 / 10$ of the Milky Way mass; to be precise, we use the term "dwarfs" here to identify galaxy with mass below that value.

If most of the IGM metals by $z=6$ have been ejected from dwarf galaxies at high redshifts, such early enrichment scenario has two important implications, that we discuss in turn.

\subsection{The filling factor argument}

First, the volume filling factor of enriched material, quantified by the porosity factor, $Q$, becomes large if pollutants are dwarf galaxies. This can be seen as follows. In a $\Lambda \mathrm{CDM}$ universe, structure formation is a hierarchical process in which nonlinear, massive structures grow via the merger of smaller initial units. Large numbers of low-mass galaxy halos are expected to form at early times in these popular cosmogonies, perhaps leading to an era of widespread pre-enrichment and preheating. The Press-Schechter (hereafter PS) theory for the evolving mass function of dark matter halos predicts a power-law dependence, $d N / d \ln m \propto m^{\left(n_{\text {eff }}-3\right) / 6}$, where $n_{\text {eff }}$ is the effective slope of the CDM power spectrum, $n_{\mathrm{eff}} \approx-2.5$ on subgalactic scales. As hot, metal-enriched gas from SN-driven 
winds escapes its host halo, shocks the IGM, and eventually forms a blast wave, it sweeps a region of intergalactic space which increases with the $3 / 5$ power of the energy $E$ injected into the IGM (in the adiabatic Sedov-Taylor phase). The total fractional volume or porosity, $Q$, filled by these 'metal bubbles' per unit explosive energy density $E d N / d \ln m$ is then

$$
Q \propto E^{3 / 5} d N / d \ln m \propto(d N / d \ln m)^{2 / 5} \propto m^{-11 / 30} .
$$

Within this simple scenario it is the star-forming objects with the smallest masses which will arguably be the most efficient pollutant of the IGM on large scales.

\subsection{The cooling argument}

The second point concerns the ability of the shocked gas to cool. This is necessary as at $z=3-3.5$, Ly $\alpha$ clouds show a spread of at most an order of magnitude in their metallicity, and their narrow line widths require that they be photoionized and cold rather than collisionally ionized and hot. At these redshifts, hot rarefied gas, exposed to a metagalactic ionizing flux, will not be able to radiatively cool within a Hubble time. The simple formula below, gives the redshift span required for a gas of primordial composition (at the metallicities present in the $\operatorname{Ly} \alpha$ forest contribution from metal cooling is virtually negligible) at the mean cosmic density heated at some redshift $z_{i}$ to cool down:

$$
\Delta z=231\left(\Omega_{m} h^{2}\right)^{1 / 2}\left(1+z_{i}\right)^{-3 / 2} \approx 3\left(\frac{1+z_{i}}{10}\right)^{-3 / 2} .
$$

Hence, a gas that has been shock-heated at $z_{i}=9$ will be already cooled by $z=6$, but if heating occurs at $z \leqslant 5$ the cooling time will exceed the Hubble time.

In conclusion early pre-enrichment by dwarfs offers the double advantage of a large metal filling factor and of efficient cooling of the metal enriched gas ejected by galactic outflows. While it is possible that some metals were dispersed in intergalactic space at late times, as hot pressurized bubbles of shocked wind and SN ejecta escaped the grasp of massive galaxy halos and expanded, cooling adiabatically, into the surrounding medium, such a delayed epoch of galactic super-winds would have severely perturbed the IGM (since the kinetic energy of the ejecta is absorbed by intergalactic gas), raising it to a higher adiabat and producing variations of the baryons relative to the dark matter: Ly $\alpha$ forest clouds would not then be expected to closely reflect gravitationally induced density fluctuations in the dark matter distribution, and the success of hydrodynamical simulations in matching the overall observed properties of Ly $\alpha$ absorption systems would have to be largely coincidental. $\dagger$ In contrast, the observed narrow Doppler widths could be explained if the ejection of heavy elements at velocities exceeding the small escape speed of subgalactic systems were to take place at very high redshifts.

\section{Metal ejection from dwarfs: observational support}

Several theoretical arguments and numerical simulations in the literature have shown that metals and energy are easily expelled from dwarf galaxies. This does not necessarily

$\dagger$ Assume, for example, that the chemical enrichment of intergalactic gas was due to the numerous population of Lyman-break galaxies (LBGs) observed at $z=3$. With a comoving space density above $m_{\star}+1=25.5$ of $0.013 h^{3} \mathrm{Mpc}^{-3}$ (Steidel et al. 1999), a $1 \%$ filling factor would be obtained if each LBG produced a metal-enriched bubble of proper radius equal to about $140 \mathrm{~h}^{-1} \mathrm{kpc}$. To fill such a bubble in $5 \times 10^{8} \mathrm{yr}$, the ejecta would have to travel at an average speed close to $600 \mathrm{~km} \mathrm{~s}^{-1}$, with characteristic post-shock temperatures in excess of 2 million degrees. 
imply a correspondingly high gas mass loss, i.e. the galaxy is typically able to retain some fraction of its original gas which might be as high as $50 \%$. In addition, quenching of star formation associated with the SN energy deposition (mechanical feedback) is strongly advocated in order to prevent too many stars to form at high redshift (overcooling problem). What are the observational evidences in support of such metal ejection scenario?

The first one is provided by the so-called Mass-Metallicity relation. The huge local galaxy catalog put together by the Sloan Digital Sky Survey (Panter et al. 2008) has allowed to construct the relation between the metallicity, $Z$, obtained from suitable indicators and the stellar mass, $M_{*}$, of each galaxy in the sample. Such curve shows a metallicity increase with $M_{*}$ up to a break point, located at $M_{*}=10^{10} M_{\odot}$, beyond which the curve flattens considerably. A reasonable fit to the median line can be obtained using a tanh function over the mass range that contains more than 2000 galaxies $\left(10^{8.8} M_{\odot}<M_{*}<10^{11.8} M_{\odot}\right)$ of the form:

$$
\log \left(Z / Z_{\odot}\right)=A+B \tanh \left(\frac{\log M_{*}-\log M_{c}}{\Delta}\right),
$$

where $\log M_{c}=9.66, \Delta=1.04, A=-0.45, B=0.57$ and masses are expressed in solar mass units. The behavior of the relation can be readily interpreted in the framework of the basic early enrichment picture. Low-mass galaxies are prone to metal loss and therefore their metallicity is suppressed by a factor that is inversely proportional to their mass. As the gravitational potential increases - along with the loss of coherent action of individual superbubbles in driving the outflow - moving towards more massive and larger objects, $Z$ approaches the effective yield value expected from a simple closed box model which describes well the flat trend deduced for galaxies with mass $M>10^{10} M_{\odot}$. It is noticeable that a very similar mass-metallicity relation starts to emerge from observations at higher redshifts, up to $z=3.3$ in agreement with numerical simulations which predict it to hold even at $z \approx 6$.

The second strong argument in favor of the fact that dwarf galaxies are the most likely source of the metals detected in the Ly $\alpha$ forest comes from the $M_{*}$ dependence on the galaxy host halo mass, $M_{h}$. The general trend of this relation is one in which the darkto-visible mass ratio steadily decreases as a function of $M_{*}$ from the smallest galaxies in the sample up to $M_{*} \approx 10^{10.5} M_{\odot}$ and then remains essentially constant above the scale. A handy fit to the $M_{*}-M_{h}$ curve can be cast in the following functional form:

$$
M_{*}=2.3 \times 10^{10} M_{\odot} \frac{\left(M_{h} / 3 \times 10^{11} M_{\odot}\right)^{3.1}}{1+\left(M_{h} / 3 \times 10^{11} M_{\odot}\right)^{2.2}} .
$$

The interpretation of this behavior is quite straightforward. Dwarf galaxies have typical dark-to-visible mass ratios $\left(M_{h} / M_{*}\right)>30$, a value largely exceeding the cosmological value $\Omega_{M} / \Omega_{b} \approx 6$, as a substantial amount of their gas has been ejected (along with their metals), whereas Milky Way-size galaxies have been able to retain most of their baryons and evolve in an almost closed-box mode.

These two evidences hence represent very strong arguments in favor of the dominant role played by dwarf galaxies in the metal enrichment process, which occurred along with reionization during the first billion year. The next question concerns when metals were mostly injected in the IGM and their specific abundance evolution. To answer these questions we need to resort a combination of data and theory, as explained in the next Section. 


\section{Metal enrichment evolution}

A growing evidence has been accumulating in the last decade from the analysis of metal absorption line spectra of high redshift $(2<z<6)$ quasars that the evolution of the most readily observed species as $\mathrm{C}$ IV and Si IV is close to flat. This result is particularly surprising since C IV abundance depends on both the overall carbon abundance and its ionization fraction into $\mathrm{C}$ IV, which in turn depends on the evolution of the UV background intensity. Fig. 1 shows a compilation of the data points showing the evolution of $\Omega_{C I V}$ together with the predicted behavior of the metallicity with redshift adopted from Scannapieco, Ferrara \& Madau (2002). Apart from the uncertain conversion between $\mathrm{C}$ IV and $C$ abundance, and the assumption of solar metallicity abundances in order to obtain the value of IGM metallicity $Z$, the striking flatness of the data is very well reproduced by the model. The ability of the model to reproduce such feature is due to the implementation of the prescription eq. 2.8 into the hierarchical build up of galaxies. The result is that in the initial phases of structure formation the most common objects (say $2 \sigma$ fluctuations of the density field) are dwarfs that are able to spread their metals; hence the metallicity grows rapidly. However, when the characteristic mass scales shift into the regime of "normal" galaxies for which $\delta_{B} \rightarrow 0$, metals are retained within the potential well of the galaxy and the metallicity levels off to an almost constant level. One critical point concerning this explanation has to do with the fact that the UV background ionizing radiation changes by a factor $\approx 10$ between $z=2$ and $z=6$. Yet, even assuming that $Z$ remains constant, one would expect that the abundances of ionized species like

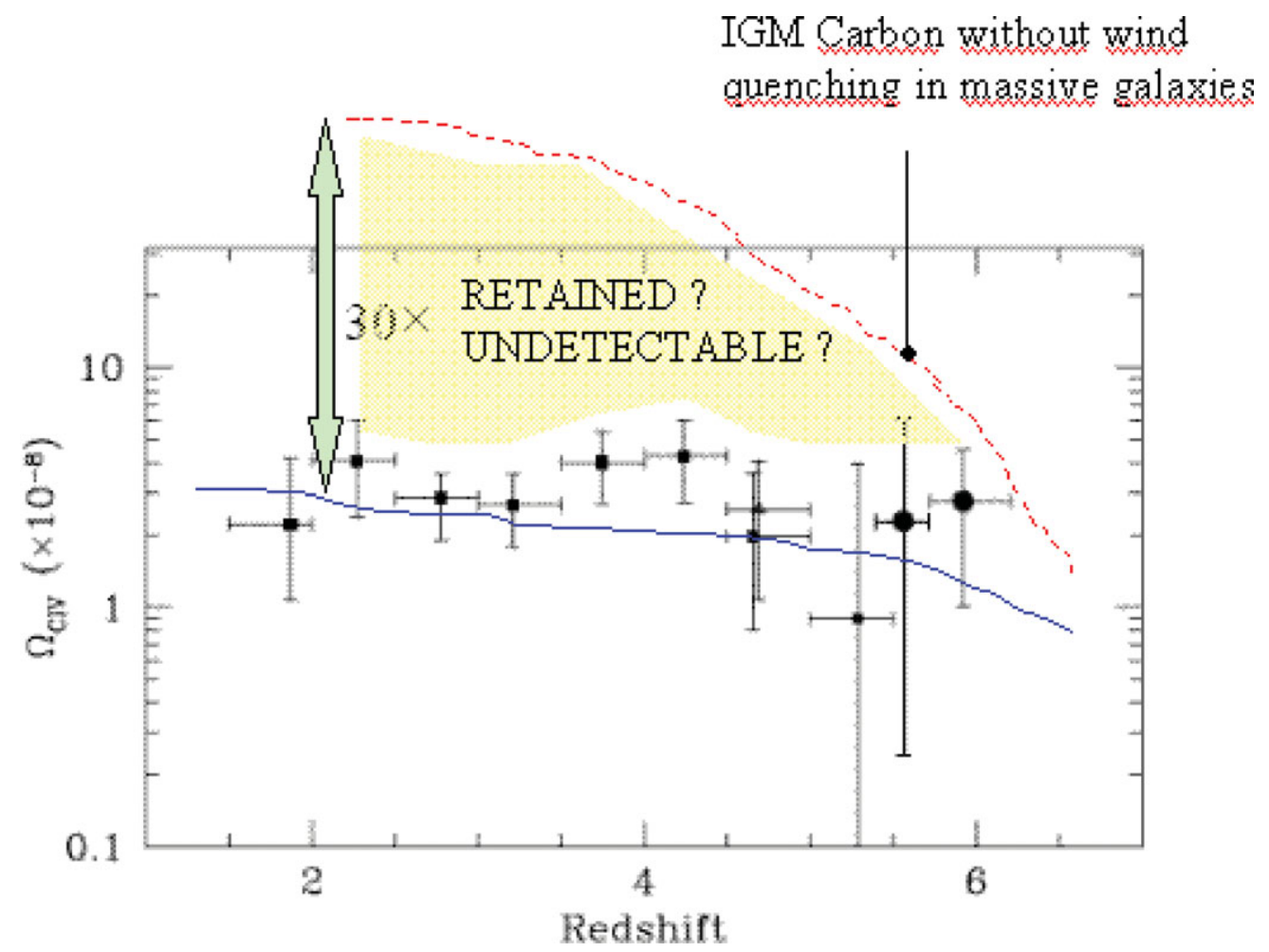

Figure 1. Data compilation for the redshift evolution of $\Omega_{C I V}$ (points) superposed to the theoretical prediction by Scannapieco, Ferrara \& Madau (2002) shown by the continuous line. The dotted line shows the evolution of the same quantity without accounting for the wind quenching in massive galaxies (see text). 
$\mathrm{C}$ IV and Si IV would change as a consequence of the different photoionization rate to which they are exposed. Nothing similar is observed. This might advocate a changing ionization correction which conspires to balance the UVB evolution to yield the flat redshift dependence observed. Such huge correction (as high as a factor of 30) can be achieved by collisional ionizations if the gas is hot, with temperatures of several $10^{5} \mathrm{~K}$. Although this is generally possible, it might cause difficulties related to an overproduction of species like $\mathrm{O}$ VI ; in addition, and more worrying, it might broaden the absorption lines to widths that exceed by far the observed ones. An alternative possibility is that the metal ionization is not dominated by the UVB but rather by a proximity of nearby star forming galaxies. There is clear evidence for clustering of metals (Pichon 2003) in regions that are typically $2-3$ comoving $\mathrm{Mpc}$ away from large galaxies $\left(M \approx 10^{12} M_{\odot}\right)$ and therefore it is likely that within this region the flux from the galaxy is dominating over the UVB one. Note that the such clustering does not imply that metals have been ejected from the large galaxy itself at the redshift at which they are observed $(z=3-4)$, but they could have been produced earlier by similarly biased (but low-mass) galaxies. Equally good fits to the clustering distribution of all observed ionized species are found in a similarly biased high-redshift enrichment model in which metals are placed within 2.4 comoving $\mathrm{Mpc}$ of $3 \times 10^{9} M_{\odot}$ sources at $z=7.5$.

\section{A viable picture}

Based on the previous considerations we propose a global scenario, illustrated schematically in Fig. 2, for the metal enrichment which is consistent with all the observations and physical processes than need to be accounted for and included in the model. Such scenario predicts that the IGM is a two-phase medium, with a hot phase at a temperature of about a million degree $\mathrm{K}$ and a cooler one with $T$ of the order of several $10^{4} \mathrm{~K}$. The hot phase is constituted by gas that is shock-heated by supernova-driven wind from the most active galaxies at redshift $z=3-5$; these galaxies are likely to be identified with the LBGs. This gas contains freshly synthesized metals, and it is located within galactic halos, thus in bubbles with comoving sizes $<1 \mathrm{Mpc}$; due to their high temperatures, low filling factor, and collisional ionization corrections, these metals hardly appear in UV/optical QSO absorption studies using O vi (let alone C IV and Si IV). The colder, photoionized component instead contains metals that were produced at higher redshifts by the dwarf galaxy population. As a result, their filling factor is about 10 times larger than the hotter component and it is much easier to detect. This simple scenario emerges from all the data discussed above, resulting in a viable metal enrichment picture. However, it can make additional predictions that concern the so-called "missing metals" problem that we are going to discuss next.

\section{The missing metals}

In its original formulation (Pettini 1999), the "missing metals" problem was stated as follows. Studies of the comoving luminosity density of distant galaxies allow us to trace the cosmic star formation density (or history, SFH), $\dot{\rho}_{\star}(z)$, up to redshift $z_{\max } \approx 7$. Assuming an initial mass function of such stars (IMF), one can compute the specific fraction of heavy elements ('metals') they produce, $y$, and derive the metal production rate $\dot{\rho}_{Z}(z)=y \dot{\rho}_{\star}(z)$, whose integral from $z_{\text {max }}$ gives the density of cosmic metals in units of the critical density, $\Omega_{Z}^{s f h}$, at any given $z$. Early searches in cosmic structures for 
which the metal/baryon mass ratioł can be derived either via intergalactic gas quasar absorption line experiments (Damped Ly $\alpha$ Absorbers [DLAs] or the Ly $\alpha$ 'forest') or through direct spectroscopic studies (Lyman Break Galaxies [LBGs]) have found that only $\Omega_{Z}^{o b s} \lesssim 0.20 \Omega_{Z}^{s f h}$ is stored in these components, i.e. the large majority of the metals are 'missing'. An analogous missing metal problem is also found by considering in a self-consistent manner the star formation rates and metallicities of DLAs alone (Wolfe et al. 2003, Prochaska et al. 2003). Newly available high-quality data allow a more precise analysis of the problem.

To re-evaluate $\Omega_{Z}^{s f h}$, Ferrara, Scannapieco \& Bergeron (2005) use a recent SFH compilation (Bouwens et al. 2005) corrected upwards for the effects of dust obscuration by the prescribed (Reddy \& Steidel 2004) value of 4.5 at $z \approx 3$ and adopt $y=1 / 42$. Integration of $\dot{\rho}_{Z}(z)$ to $z=2.3$ yields $\Omega_{Z}^{s f h}=1.84 \pm 0.34 \times 10^{-5}$. Where should we look for these metals?

The most obvious locations are the galaxies used to derive the SFH, i.e. LBGs. These are characterized (Reddy \& Steidel 2004) by a mean comoving number density of $6 \times 10^{-3} h^{3} \mathrm{Mpc}^{-3}$ and $Z=0.6 Z_{\odot}=0.0113$. Stellar masses can be constrained only by assuming a range of star formation histories of the form $S F R(t) \propto \exp (-t / \tau)$ and therefore they are somewhat uncertain. According to Shapley et al. (2005), they should be in the range $0.6-6 \times 10^{10} M_{\odot}$. Assuming the best fit value $M_{\star}=2 \times 10^{10} M_{\odot}$, we get $\Omega_{Z}^{l b g}=3.4 \times 10^{-6} M_{\star, 10} \approx 0.18 \Omega_{Z}^{s f h}$. If metals are not in LBG stars or gas, they could be in DLAs or the IGM. The metal content of DLAs is derived by noting that (Rao \& Turnsheck 2000, Prochaska \& Wolfe 2000$)$ at $z \approx 3$ their neutral $\left(\approx\right.$ total) gas density $\Omega_{g}^{\text {dla }}=10^{-3}$ and metallicity $Z=3.8 \times 10^{-4}$ combine to give $\Omega_{Z}^{\text {dla }}=3.8 \times 10^{-7}<\Omega_{Z}^{\text {obs }} \ll \Omega_{Z}^{\text {sfh }}$; we can therefore neglect their contribution. In the following, we correct for LBG contribution by (re)defining the cosmic density of missing metals $\Omega_{Z}^{s f h} \equiv \Omega_{Z}^{s f h}-\Omega_{Z}^{l b g}$.

Hence, the missing metals should be found outside the main body of galaxies or DLAs. There are essentially two possibilities: (a) they could reside in the Ly $\alpha$ forest, or (ii) in the halos of the galaxies that have produced and ejected them. Note that the distinction between these two components is somewhat ambiguous. Our working definition is that

$\dagger$ We adopt a solar metallicity $Z_{\odot}=0.0189$ by mass, and the notation $Y_{x}=Y / 10^{x}$

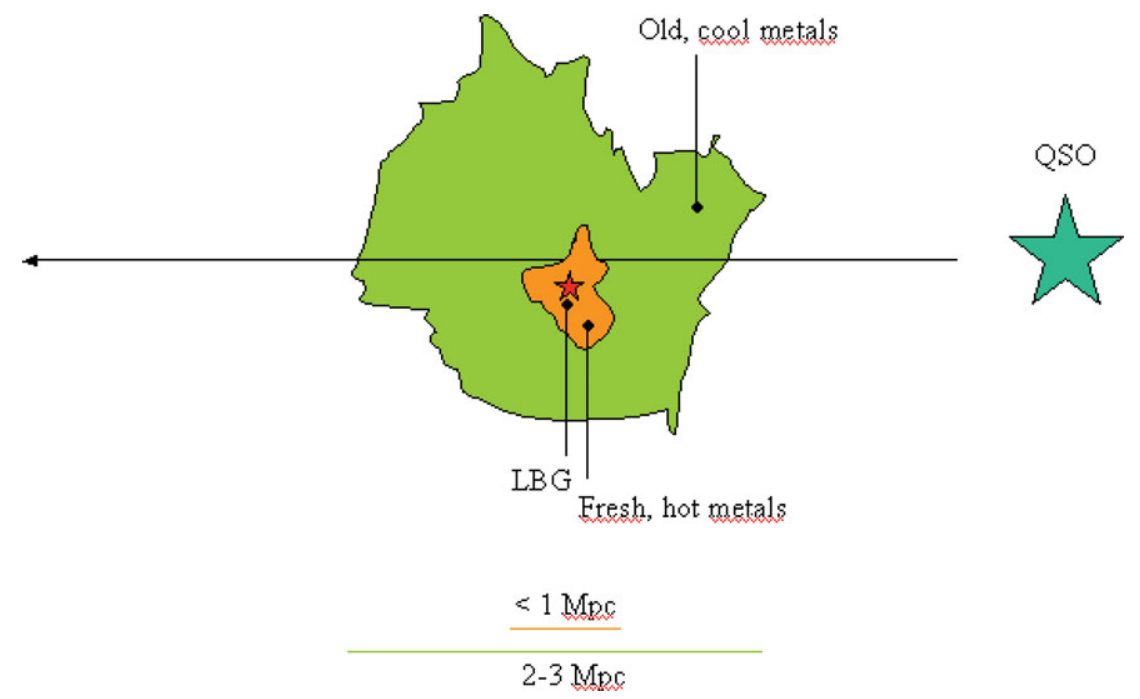

Figure 2. Sketch of the proposed two-phase IGM metal enrichment scenario 


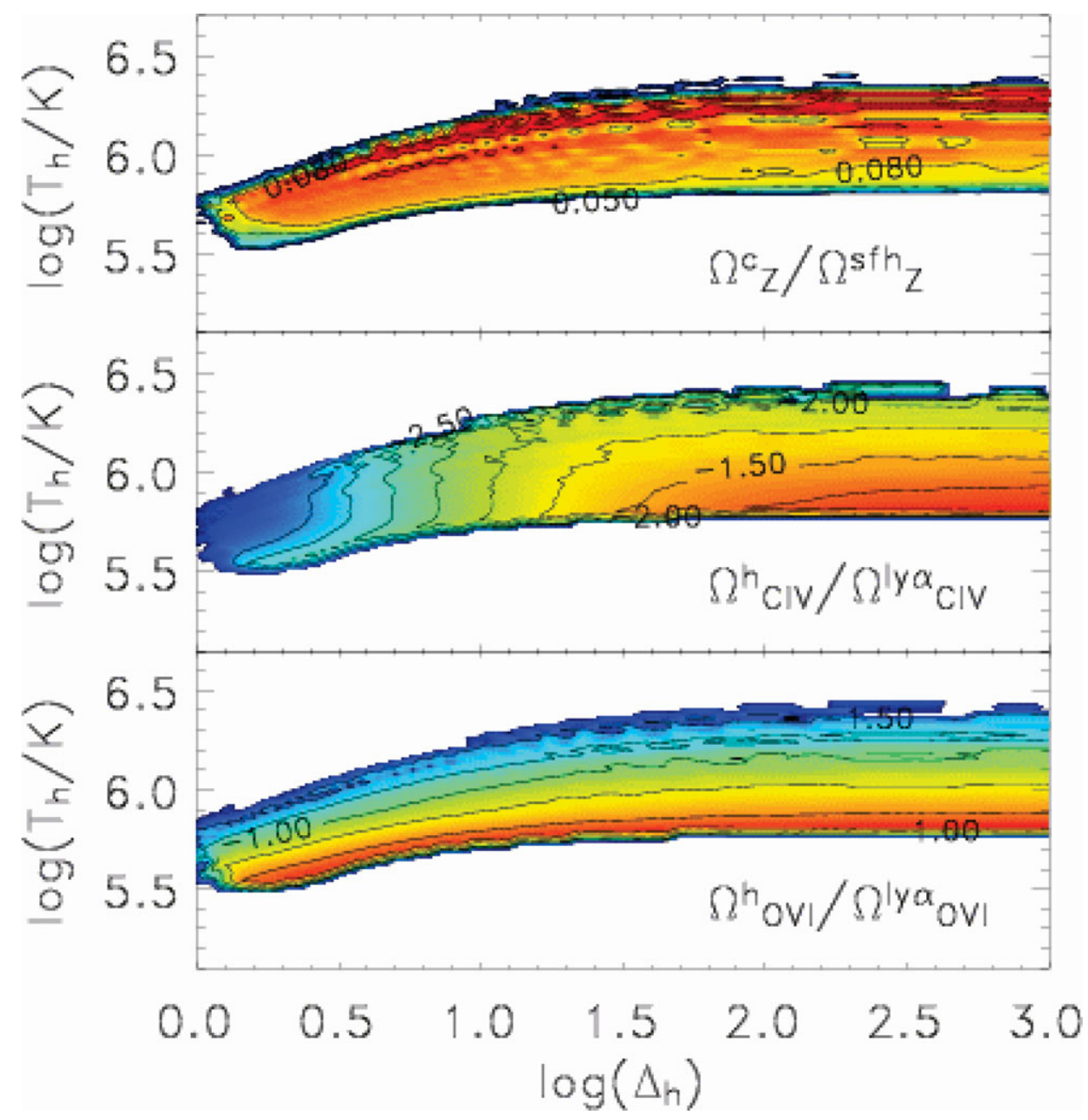

Figure 3. Allowed hot phase gas temperature, $T_{h}$, and overdensity, $\Delta_{h}$, regions. Also shown are the corresponding isocontours of the $\Omega_{Z}^{c} / \Omega_{Z}^{s f h}$ (upper panel), $\Omega_{\mathrm{CIV}}^{h} / \Omega_{\mathrm{CIV}}^{l y \alpha}$ (middle panel) and $\Omega_{\mathrm{OVI}}^{h} / \Omega_{\mathrm{OVI}}^{l y \alpha}$ (bottom panel).

galactic halos are gravitationally bound structures around galaxies; they are special sites affected by galactic winds.

The most studied tracers of $\operatorname{Ly} \alpha$ forest metallicity are C IV and O vi absorption lines. The fraction of the critical density $\rho_{c}$ contributed by a given element ion, $E_{i}$, of mass $m_{E}$ residing in the Ly $\alpha$ forest is given by

$$
\Omega_{E_{i}}^{l y \alpha}=\frac{H_{0}}{\rho_{c} c} \frac{\sum N_{E_{i}}}{\sum \Delta X} m_{E}
$$

where $\Delta X\left(z_{+}, z_{-}\right)=\int_{z_{-}}^{z_{+}} d z(1+z)^{2} E(z)^{-1}$ is the absorption distance (Bahcall \& Peebles 1969), with $E(z)=\left[\Omega_{\Lambda}+\Omega_{m}(1+z)^{3}\right]^{1 / 2}$; sums are performed over all the redshift intervals $z_{-}<z<z_{+}$over which an ion column density $N_{E_{i}}$ is detected. To determine $\Omega_{\mathrm{OVI}}^{\text {ly } \alpha}$ and $\Omega_{\mathrm{CIV}}^{\text {ly } \alpha}$, we use data from the ESO VLT Large Program (Bergeron et al. 2002) which provides high $\mathrm{S} / \mathrm{N}$, high resolution spectra of an homogeneous 
sample of 19 QSOs in $1.7<z<3.8 ; \Omega_{\mathrm{OVI}}^{\text {ly } \alpha}$ is currently available for four LP lines of sight (Bergeron et al. 2002), for which we find $\Omega_{\mathrm{OVI}}^{l y \alpha}=1.3 \times 10^{-7}$; two other recent estimates (Simcoe, Sargent \& Rauch 2004, Carswell, Schaye \& Kim 2002), give $\Omega_{\text {OVI }}^{\text {ly }}=(1.1,0.9) \times 10^{-7}$. We adopt the sightline-weighted mean of the three values allowing for the largest error, $\Omega_{\mathrm{OVI}}^{\text {ly }}=1.1 \pm 0.3 \times 10^{-7}$. From the $\mathrm{C}$ IV absorber distribution (Aracil et al. 2004, Scannapieco et al. 2005) in the column density range $12<\log N_{\mathrm{CIV}}<16$ we obtain $\Omega_{\mathrm{CIV}}^{l y \alpha}=7.5 \pm 2.2 \times 10^{-8}$ (statistical error). This value is about two times higher than previous determinations (Songaila 2001; Simcoe, Sargent \& Rauch 2004, Schaye et al. 2003) which could not account for the contribution of strong $(\log \mathrm{C}$ IV $>14)$ absorption systems. Combining the average measured $N_{\mathrm{CIV}}-N_{H}$ I and $N_{\mathrm{OVI}}-N_{H I}$ correlations (Aracil et al. 2004) with the measured distribution of weak $H I$ absorbers (Petitjean et al. 1993), we have checked that systems with $\log N_{\mathrm{CIV}}<12$ contribute less than 1\%, well within the quoted error. For a (meteoritic) solar carbon logarithmic abundance (in number) $A_{C}=8.52$ with respect to hydrogen $\left(A_{H}=12\right.$ ), we conclude that only a fraction $\Omega_{\mathrm{CIV}}^{l y \alpha} / \Omega_{C}^{s f h}=2.4 \times 10^{-2}$ of the produced carbon is observed in the $\mathrm{C}$ IV state. Repeating the procedure for $\mathrm{O}\left(A_{O}=8.83\right)$, gives a ratio $\Omega_{\mathrm{OVI}}^{l y \alpha} / \Omega_{O}^{s f h}=1.3 \times 10^{-2}$. To account for all uncertainties above, we will consider values in the range $1.4 \times 10^{-2}<\Omega_{\mathrm{CIV}}^{\text {ly }} / \Omega_{C}^{s f h}<4.0 \times 10^{-2}$ and $8.1 \times 10^{-3}<\Omega_{\mathrm{OVI}}^{\text {ly } \alpha} / \Omega_{O}^{s f h}<2.1 \times 10^{-2}$. We now determine the physical conditions of the gas hiding the missing $\mathrm{C}$ and O. Numerical simulations (Davé et al. 2001) suggest that the intergalactic medium [IGM] might be a two-phase system made by a cool $\left(T_{c} \approx 10^{4-4.5} \mathrm{~K}\right)$, photoionized phase, and a hot, collisionally ionized one. We impose the following conditions separately for each ion ( $\mathrm{C}$ IV,O VI ) and element (C,O): (1) the observed ionic abundance is the sum of the abundances in the two phases; (2) the SFH-produced element abundance is the sum of the element abundances in the two phases; (3) the elements are in the same abundance ratios in the two phases. More explicitly, these conditions can be mathematically expressed as

$$
\begin{aligned}
f_{C}^{c} \Omega_{C}^{c}+f_{C}^{h} \Omega_{C}^{h} & =\Omega_{\mathrm{CIV}}^{l y \alpha} \\
f_{O}^{c} \Omega_{O}^{c}+f_{O}^{h} \Omega_{O}^{h} & =\Omega_{\mathrm{OVI}}^{l y \alpha} \\
\Omega_{C}^{c}+\Omega_{C}^{h} & =\Omega_{C}^{s f h} \\
\Omega_{O}^{c}+\Omega_{O}^{h} & =\Omega_{O}^{s f h} \\
\Omega_{C}^{c}-A \Omega_{O}^{c} & =0 \\
\Omega_{C}^{h}-A \Omega_{O}^{h} & =0
\end{aligned}
$$

After some simple algebra, the above equations reduce to:

$$
\frac{\Omega_{\mathrm{CIV}}^{l y \alpha} / \Omega_{C}^{s f h}-f_{C}^{h}}{f_{C}^{c}-f_{C}^{h}}=\frac{\Omega_{\mathrm{OVI}}^{l y \alpha} / \Omega_{O}^{s f h}-f_{O}^{h}}{f_{O}^{c}-f_{O}^{h}},
$$

where $f_{i}^{j} \equiv f_{i}^{j}\left(\Delta_{j}, T_{j}, \mathcal{U}_{j}\right)$ is the ionization correction for the considered ion ( $\mathrm{C}$ IV or $\mathrm{O}$ VI $)$ of a given element $(i=C, O)$ in the cold or hot phase, $(j=c, h)$, respectively; the overdensity, $\Delta_{j}$ and temperature, $T_{j}$, of the two phases are the unknowns of the problem; finally, $A$ is the abundance ratio of the two elements. We complement these conditions by further imposing that the pressure of the cool phase does not exceed that of the hot one and assuming a temperature-density relation for the cold phase $T=T_{0} \Delta_{c}^{\gamma}$, (with $T_{0}=2 \times 10^{4} \mathrm{~K}$ and $\gamma=0.3$ ), as inferred from the Ly $\alpha$ forest data. The value of the 

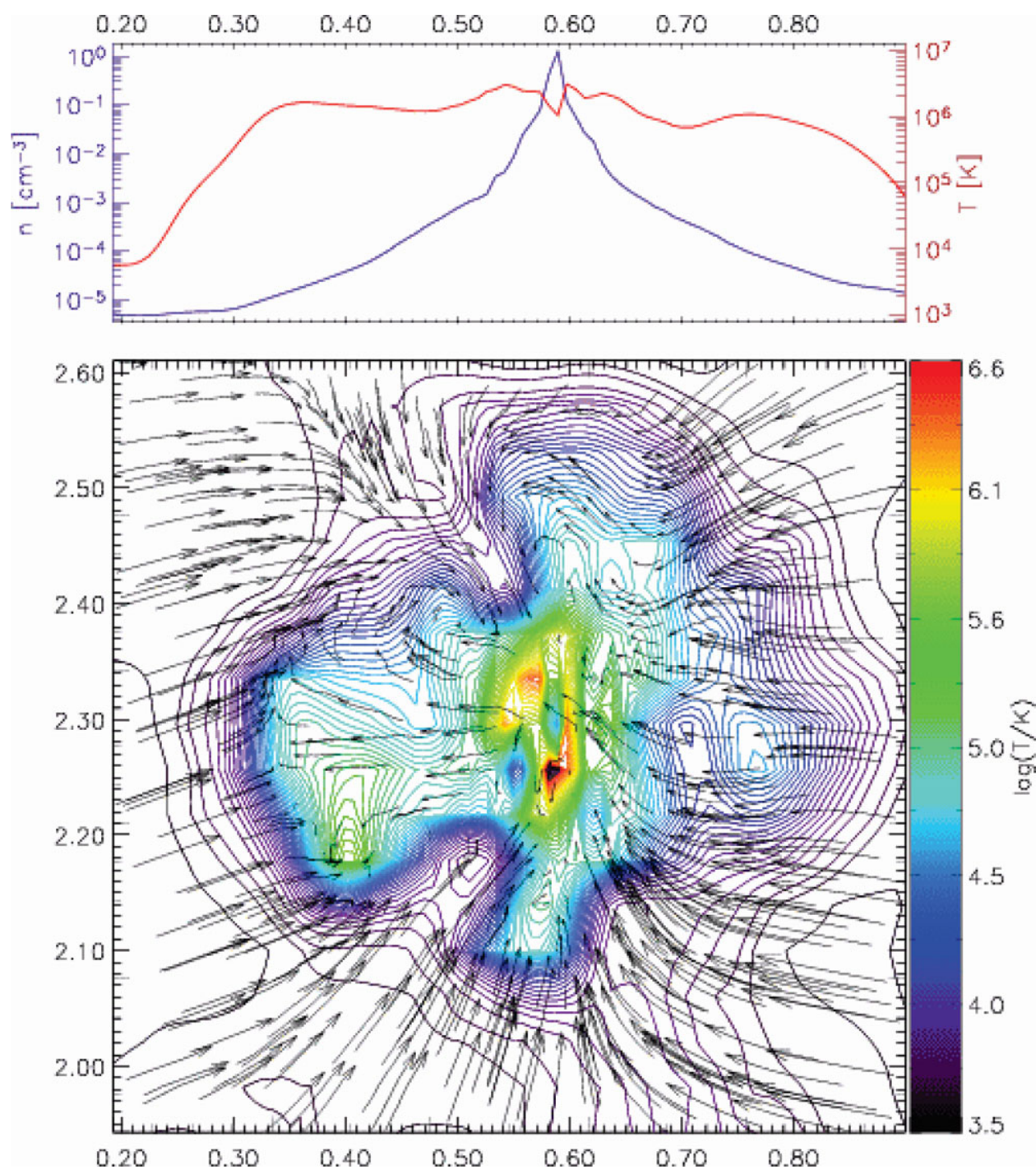

Figure 4. Bottom panel: Temperature map (physical Mpc units) of a plane through the center of a simulated starburst galaxy at $z=3.3$ with properties typical of Lyman Break Galaxies; longest velocity vectors correspond to $v=150 \mathrm{~km} \mathrm{~s}^{-1}$. Upper panel: $1 \mathrm{D}$ cuts parallel to the horizontal axis and passing through the center of the map in the bottom panel showing the gas density (blue) and temperature (red).

photoionization parameter, $\mathcal{U}_{j}=n_{\gamma} / n_{j}$, is fixed by the ionizing photon density $n_{\gamma}$ of the assumed UV background spectrum (Haardt \& Madau 1996) shifted so that the intensity at 1 Ryd is $J_{\nu}=0.3 \times 10^{-21} \mathrm{erg} \mathrm{s}^{-1} \mathrm{~Hz}^{-1}=0.3 J_{21}$, corresponding to a hydrogen photoionization rate $\Gamma=0.84 \times 10^{-12} \mathrm{~s}^{-1}=0.84 \Gamma_{12}$, in agreement with Bolton et al. (2005). Finally, we warn that deviations from solar abundances might be possible, and indeed there are hints that oxygen might be overabundant (Telfer et al. 2002; Bergeron et al. 2002); here we neglect this complication.

By solving eq. 6.8, we obtain the results plotted in Fig. 3. The hot phase is characterized by a wide density range, $\log \Delta_{h}>0.4$ and a restricted temperature range $5.8<\log T_{h}<$ 6.4. We find that: (i) only $5 \%-9 \%$ of the produced metals reside in the cold phase, the 
rest being found in the hot phase; (ii) $1 \%-6 \%(3 \%-30 \%)$ of the observed $\mathrm{C}$ IV (O VI ) is in the hot phase. We conclude that more than $90 \%$ of the metals produced during the star forming history can be placed in a hot phase of the IGM, without violating any observational constraint. To further constrain the hot phase parameter range, we have searched in the LP C IV line list for components with large Doppler parameters. We find no lines with $b_{\mathrm{CIV}} \geqslant 26.5 \mathrm{~km} \mathrm{~s}^{-1}$, corresponding to $\log T_{h}>5.7$; this result seems to exclude the high density and temperature region of the allowed parameter space in the middle panel of Fig. 3. We checked that the above findings are insensitive to variations of $\Gamma_{12}$ of $\pm 50 \%$; however, O VI /C IV ratios in the cold phase might depend on the UVB shape around 4 Ryd.

The derived values of $T_{h}$ and $\Delta_{h}$ are suggestive of regions likely to be found around galaxies; moreover, $10^{6} \mathrm{~K}$ gas temperature would have a scale height of $>10 \mathrm{kpc}$, hence it cannot be confined in the disk. To test this hypothesis we resort to cosmological simulations. As an illustration, Fig. 4 shows the temperature and velocity structure in a 2D cut through the center of a simulated galaxy (we used the multiphase version [Marri \& White 2003] of the GADGET2 code to simulate a comoving $10 h^{-1} \mathrm{Mpc}^{3}$ cosmic volume) at redshift $z=3.3$; its total (dark + baryonic) mass is $2 \times 10^{11} M_{\odot}$, the star formation rate $\approx 20 M_{\odot} \mathrm{yr}^{-1}$. This galaxy has been selected to match LBG properties, but it is not unusual in the simulation volume. As often observed in LBGs, a strong galactic wind is visible, whose expansion is counteracted by energy losses due to cooling and gravity, and ram pressure exerted by the infalling gas. Infall is particularly effective at confining the wind into a broadly spherical region of physical radius $\approx 300 \mathrm{kpc}$, into which cold infalling streams of gas penetrate. Inside such wind-driven bubble the temperature (Fig. 2) is roughly constant $T \approx 10^{6} \mathrm{~K}$, whereas the density spans values of $0<\log \Delta<5$ $\left[\Delta(z=3.3)=1\right.$ corresponds to $\left.\approx 2 \times 10^{-5} \mathrm{~cm}^{-3}\right]$. The cool phase is evident in the outer boundary of the bubble, where cooling interfaces arise from the interaction with infalling streams. Hence halos of LBGs seem to meet the requirements as repositories of missing metals.

Additional support for this conclusion comes from studies of the correlation properties of C IV and O vi absorbers (Pichon et al. 2003, Aracil et al. 2004), which conclude that: (i) $\mathrm{O}$ vi absorption in the lowest density gas is usually (about $2 / 3$ of the times) located within $\approx 300-400 \mathrm{~km} \mathrm{~s}^{-1}$ of strong $\mathrm{H}$ I absorption lines; (ii) the $\mathrm{C}$ IV correlation function is consistent with metals confined within bubbles of typical (comoving) radius $\approx 1.4 h^{-1} \mathrm{Mpc}$ in halos of mass $M \geqslant 5 \times 10^{11} M_{\odot}$ at $z=3$. If each of such objects hosts one bubble, the cosmic volume filling factor of metals is $f_{Z}=11 \%$; it follows that halo metallicity is $\Omega_{Z}^{s f h} / f_{Z} \Omega_{b}=0.165 Z_{\odot}$. A temperature of $\log T_{h}=5.8$ corresponds to $\mathrm{H}$ I $(\mathrm{O}$ vI $)$ Doppler parameters $b_{\mathrm{HI}}=102\left(b_{\mathrm{OVI}}=25.5\right) \mathrm{km} \mathrm{s}^{-1}$ and to $N_{\mathrm{OVI}} / N_{\mathrm{HI}}=3$; absorbers with $\log N_{\mathrm{OVI}}=13$ are detectable for $b_{\mathrm{OVI}}=25.5 \mathrm{~km} \mathrm{~s}^{-1}$ but the corresponding $\log N_{\mathrm{HI}}=12.4$ ones for $b_{\mathrm{HI}}=102 \mathrm{~km} \mathrm{~s}^{-1}$ are not. This raises the possibility of finding $\mathrm{O}$ vi absorbers without associated H I .

\section{Implications}

The scenario proposed leads to several interesting consequences. First, metals produced by LBGs do not seem to be able to escape from their halos, due to the confining mechanisms mentioned above. This is consistent with the prediction (Ferrara, Pettini \& Shchekinov 2000) that galaxies of total mass $\mathcal{M}>10^{12}(1+z)^{-3 / 2} M_{\odot}$ do not eject their metals into the IGM. Interestingly, the metallicity-mass relation recently derived from the SDSS (Tremonti et al. 2004) shows that galaxies with stellar masses above $3 \times 10^{10} M_{\odot}$ (their total mass corresponds to $\mathcal{M}$ for a star formation efficiency $f_{\star}=0.2$ ) 
chemically evolve as "closed boxes," i.e. they retain their heavy elements. Second, the nearly constant $\left(2 \leqslant z \leqslant 5, Z \approx 3.5 \times 10^{-4} Z_{\odot}\right)$ metallicity of the low column density IGM (Songaila 2001) is naturally explained by the decreasing efficiency of metal loss from larger galaxies. Early pollution from low-mass galaxies allows a sufficient time for metals to cool after ejection; however, the majority of metals in LBG halos at lower redshifts are still too hot to be detected. Hence their contribution to the metallicity evolution of the IGM cannot be identified by absorption line experiments, which mostly sample the cool phase of the forest. Third, the rapid deceleration of the wind results either in a quasi-hydrostatic halo or in a 'galactic fountain' if radiative losses can cool the halo gas. In both cases this material is very poorly bound and likely to be stripped by ram pressure if, as it seems reasonable, the galaxy will be incorporated in the potential well of a larger object (galaxy group or cluster) corresponding to the next level of the hierarchical structure growth. Turbulence and hydrodynamic instabilities associated with this process are then likely to efficiently mix the metals into the surrounding gas within approximately a sound crossing time of $\sim 1 \mathrm{Gyr}$, or $\Delta z \approx 0.5$. If metals produced and stored in LBG halos by $z=2.3$ end up in clusters, than the average metallicity of the intracluster medium is $Z_{I C M}=\Omega_{Z}^{s f h} / \Omega_{I C M}=0.31 Z_{\odot}$, having assumed (Fukugita, Hogan \& Peebles 1998) $\Omega_{I C M}=0.0026 h_{70}^{-1.5}$. Not only is this number tantalizingly close to the observed value at $z=1.2$ (Tozzi et al. 2003), but we also predict that little evolution will be found in the ICM metallicity up to $z \approx 2$ as essentially all the metals that could have escaped galaxies during cosmic evolution had already done so by this epoch.

\section{References}

Aracil, B., Petitjean, P., Pichon, C. \& Bergeron, J. 2004, A\&A, 419, 811

Bahcall, J. \& Peebles, J. 1969, ApJ, 156, L7

Bergeron, J., Aracil, B., Petitjean, P. \& Pichon, C. 2002, A\&A, 396, L11

Bergeron, J. \& Herbert-Fort, S. 2005, astro-ph/0506700

Bolton, J. S., Haehnelt, M. G., Viel, M., Springel, V. 2005, MNRAS, 257, 1178

Bouwens, R. J. et al. 2004, astro-ph/0409488 (2004)

Carswell, B., Schaye, J. \& Kim, T.-S. 2002, ApJ, 578, 43

Davé et al. 2001, ApJ, 552, 473

Ferrara, A., Pettini, M. \& Shchekinov, Y. 2000, MNRAS, 319, 539

Fukugita, M., Hogan, C. J. \& Peebles, P. J. E. 1998, ApJ, 503, 518

Haardt, F. \& Madau, P. 1996, ApJ, 461, 20

Marri, S. \& White, S. D. M. 2003, MNRAS, 345, 561

Panter, B. et al. 2008, preprint, astro-ph/0804.3091

Petitjean, P., Webb, J. K., Rauch, M., Carswell, R. F., \& Lanzetta, K. 1993, MNRAS, 262, 499

Pettini, M. ESO Workshop, Chemical Evolution from Zero to High Redshift, 233-247 (1999)

Pichon, C., Scannapieco, E., Aracil, B., Petitjean, P., Aubert, D., Bergeron, J. \& Colombi, S. 2003, ApJ, 597, L97

Prochaska, J. X. \& Wolfe, A. M. 2000, ApJ, 533, L5

Prochaska, J. X., Gawiser, E., Wolfe, A. M., Castro, S. \& Djorgovski, S. G. 2003, ApJL, 595, L9

Rao, S. M. \& Turnshek, D. A. 2000, ApJS, 130, 1

Reddy, N. A. \& Steidel, C. C. 2004, ApJ, 603, L13

Savage, B. D. \& Semback, K. R. 1996, ARA\&A, 34, 279

Scannapieco, E., Ferrara, A. \& Madau, P. 2002, 574, 590

Scannapieco, E. et al., MNRAS, 365, 615

Schaye, J., Aguirre, A., Kim, T.-S., Theuns, T., Rauch, M. \& Sargent, W. L. W. 2003, ApJ, 596,768 
Shapley, A. E., Steidel, C. C., Erb, D. K., Reddy, N. A., Adelberger, K. L., Pettini, M., Barmby, P., Huang, J. 2005, ApJ, 626, 698

Simcoe, R. A., Sargent, W. L. W. \& Rauch, M. 2004, ApJ, 606, 92

Songaila, A. 2001, ApJ, 561, L153

Spergel, D. N. et al. 2003, ApJ, 148, 175

Telfer, R. C., Kris, G. A., Zheng, W., Davidsen, D. A. \& Tytler, D. 2002, ApJ, 579, 500

Tozzi, P., Rosati, P., Ettori, S., Borgani, S., Mainieri, V. \& Norman, C. 2003, ApJ, 593, 705

Tremonti, C. A. et al. 2004, ApJ, 613, 898

Wolfe, C. A., Gawiser, E. \& Prochaska, J. X. 2003, ApJ, 593, 235 